\title{
DETECTION ESCHERICHA COLI BACTERIA: A REVIEW OF IMAGE PROCESSING METHODS
}

\author{
Siti Farah Binti Hussin, \\ Politeknik Mersing, Johor \\ E-mail: farah@pmj.edu.my \\ Zainolrin Bin Saari \\ Politeknik Mersing, Johor \\ E-mail: zainolrin@pmj.edu.my
}

\begin{abstract}
There have been numerous chemical studies to detect Eschericha Coli Bacteria or better known as E.coli bacteria. According to (Wisuthiphaet, et al, 2019), studies using image processing to detect E.coli bacteria have been restricted. There is an urgent need to incorporate image processing into systematic reviews for the identification of E.coli in water or food samples. The E.coli detection process takes between 24 hours to 48 hours as the bacteria needs to go through the growth phase as a result of a mixture of water samples and chemical reagents and then placed in an incubator at certain temperatures and humidity. This article review several approaches for E.coli bacteria detection from standard technique, portable hybrid imaging system, hybrid smartphone and microfluidic biosensor, smartphone and paper microfluidics and goes to fibre optics. The colour change after several hours is an indicator for detecting the presence of E.coli bacteria in the water sample. Although standard techniques such as Gold Nanoparticles, Solid phase Cytometry, Viable Plate Count and Polymerase Chain Reaction (PCR) for detection the presence of E.coli, the use of this technique involving the transfer of water samples to the laboratory, trained staff, longer sample monitoring process, expensive costs and complex procedures. E.coli detection techniques are also going through a revolutionary phase as technology advances by using a hybrid technique that combines standard technique and image processing. E.coli growth monitoring and detection process is now easier as it can be viewed using a portable device, smartphone or via a website in real-time.
\end{abstract}

Keywords: E.coli bacteria, image processing, standard technique, smartphone

\section{INTRODUCTION}

Food and water are a necessity in human life and without it human beings cannot afford to live and water is an essential source of human life. This is because water is actively used in many human activities such as beverages, washing food and for cleaning purposes. In addition, human health depends on the quality of water used. There are three measures of water quality assessment, namely physical, microbial and chemical (Dunca, 2018). The microbial method is most often used to assess water quality because it is easy to use and is capable of examining water source frequently by using the simple test. Human drinking water has the potential to be contaminated by 
bacteria called organisms. These organisms are composed of Faecal Coliforms, Total Coliforms and E.coli (Odonkor, et al, 2013). Escherichia coli or better known as E.coli is a type of bacteria found in human and animal intestines (Lim, et al, 2010). The presence of E.coli in the water indicates the presence of human or animal sewage contaminants. E. coli enters through into creeks, rivers, lakes or ground water. E.coli bacteria in the water can cause various diseases. These E.coli bacteria are extremely dangerous as they can be life threatening as reported by (Rettner,2018) five deaths were reported in Arkansas, California, two deaths in Minnesota and one death in New York due to E.coli bacteria found in Romaine lettuce. Meanwhile, more than 96 people were admitted to the hospital and twenty-seven of them showed symptoms of kidney failure. According to (Almadidy, et al, 2002) early detection of E. coli bacteria can prevent the spread of more severe disease outbreaks and divides E.coli into two types, the harmless and the dangerous. This type of harmless E.coli produces vitamin K2 in the gut and serves to protect the gut from bacterial pathogen attacks. According to (Sukhumungoon, 2015), (Kabiru, et al, 2015) and (Jang, et al, 2017), Hazardous E.coli, E. coli diarrheagenic, E. coli 0157: H7 refers to E.coli that can cause human illness such as diarrhoea, vomiting, pneumonia, urinary tract infections shown as Figure 1 (Ahmed, et al, 2015). Studies of E.coli using image processing are essential as the world is moving towards Industrial Revolution 4.0 (IR4.0), the transition from man power to cyber physical system using the latest technology such as internet of things (IoT), IoT security and cloud. In addition, this study is important for the social wellbeing aspect as this study has an impact on health community.

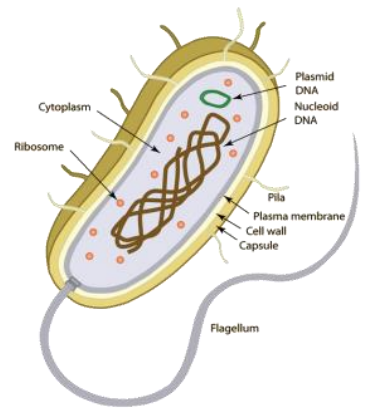

Figure 1: E.coli cell 


\section{STANDARD TECHNIQUE}

There are various techniques for detecting E.coli including Gold Nanoparticles, Solid phase Cytometry, Viable Plate Count and Polymerase Chain Reaction (PCR) as shown in Figure 2 (a) - (d). This Gold nanoparticles technique was able to detect the presence of E.coli by inductively coupled plasma mass spectrometry (ICPMS). In addition, this technique requires trained staff to control antigen targets, sensitive antigen testing and cost (Wang and Alocilja, 2015), Solid phase Cytometry uses fluorescent signals to detect viable organisms. The disadvantages of this technique is limited to the ability of detection the fluorescent signals, complicated test because it involves multiple processes and requires additional steps to detect E.coli by using a ScanRDI ${ }^{\circledR}$ device (Clark, 2001). The Viable Plate Count uses the method of calculating the bacterial concentration based on the cell count on the agar plate. Plates containing E.coli will be incubated in overnight incubators at $37^{\circ} \mathrm{C}$. However, this test has its drawbacks in requiring the implementation of the MALDI-TOF test to detect the presence of E.coli (Bajwa, et al, 2013). Meanwhile, PCR technique was invented by Kary B. Mullis in 1885 while working as a chemist at Cetus Corporation (Disotell, 2017). However, this technique has some drawbacks, require a DNA specialist, specifically for E.coli bacteria only, high cost and highly sensitive testing (Babaie, et al, 2017).

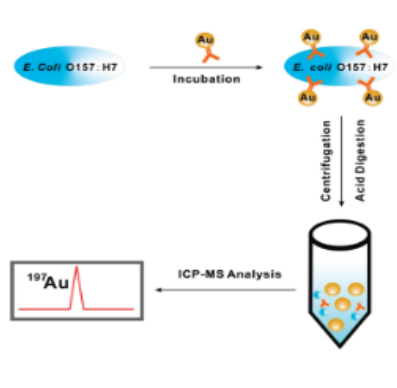

(a)

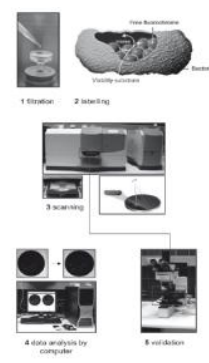

(b)

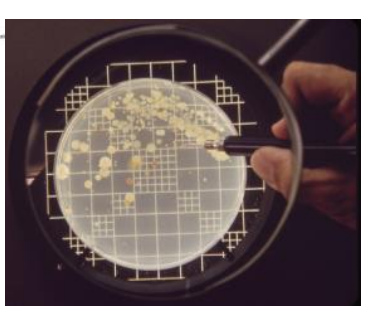

(c)

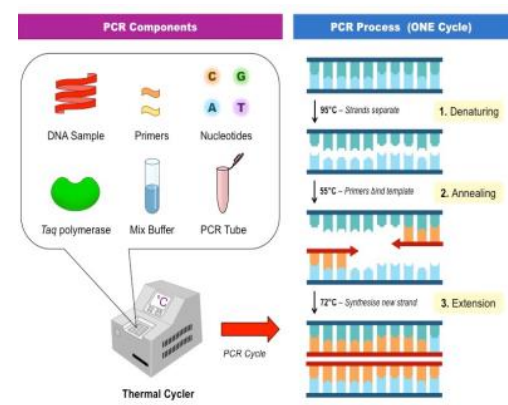

(d)

Figure 2: (a) Gold nanoparticles (b) Solid phase Cytometry

(c) Viable Plate Count (d) Polymerase Chain Reaction (PCR) 


\section{PORTABLE HYBRID IMAGING SYSTEM}

Portable hybrid imaging system for the detection of E.coli is a three-arm device that uses Surface Plasmon Resonance (SPR) imaging technology. According to (Zordan, et al, 2009), NIH ImageJ software was used to capture image foodborne illness pathogens E. coli 0157 : H7 and was able to analyze percentage area of gold spot. This method to detect E.coli's presence used a mixture of cytometry imaging device and microfluidic SPR as shown in Figure 3. Imaging cytometry device is placed on a plate reader and it is based on cell-based screening (Berney, et al, 2007) and (Lahtinen, et al, 2006). SPR is a measurement of the light intensity of the metal layer. In addition, it displays 14 pathogens in the form of positive and negative results simultaneously, portable and lightweight design makes this equipment easy to carry everywhere. It is also simple to detect E.coli as this portable hybrid imaging system will be positioned close to the sample without needing samples through an incubation method. The drawback using SPR that it can only capture bacteria if it is within the SPR range on the gold surface (Zordan, et al, 2009). This is because the detection of image epi-fluorescence is directly proportional to the SPR where the SPR is placed at the bottom of the biochip while the epi-fluorescence is at the top of the biochip (Berney, et al, 2007).

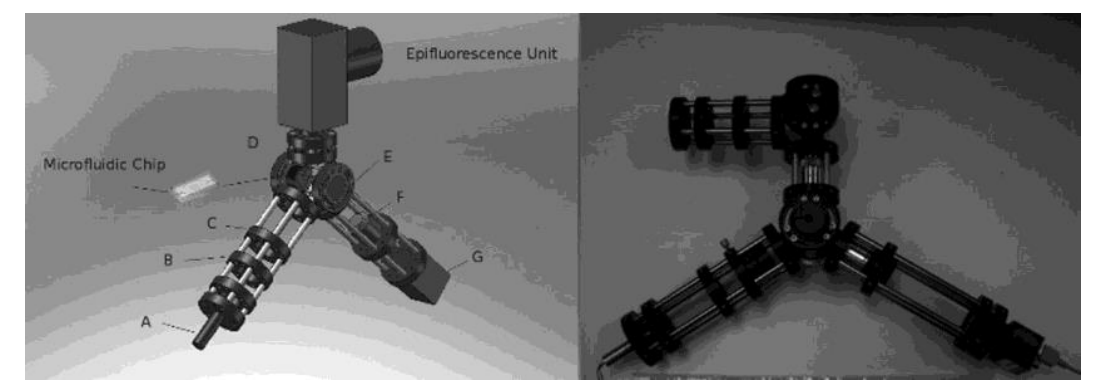

Figure 3: Portable imaging cytometry device and microfluidic SPR

\section{HYBRID SMARTPHONE AND MICROFLUIDIC BIOSENSOR}

Hybrid smartphone and microfluidic biosensor is a mix of mobile, sensor, and microfluidic component. The transition to this technology is important since prices are costly, wide and difficult to control with conventional biosensor methods $(\mathrm{Xu}$, et al, 2018). 880nm NIR LEDs with smartphones equipped with digital cameras, Apple's Xcode software that enables users to capture 4 different angles at fixed distance, 
MiePlot v4.2.11 software, a sphere-scattering computer program using Mie theory \& the Debye series and gyro sensors internally used to detect E.coli in beef (Liang, et al, 2014). This $880 \mathrm{~nm}$ NIR LED is placed perpendicular to the beef and digital cameras will detect scatter light at $15^{\circ}, 30^{\circ}, 40^{\circ}$ and $60^{\circ}$ angles using positioning stages as shown in Figure 4 (a). Figure 4 (b) uses a smartphone-based detection system at $15^{\circ}$, $30^{\circ}, 40^{\circ}$ and $60^{\circ}$ angles without using positioning stages by programming the distance and angle between smartphones to ground beef in Xcode Apple software and adjusting the NIR LED together with built-in gyro sensor. Figure 4 (c) shows the actual picture taken using a smartphone connected to an 880nm NIR LED.

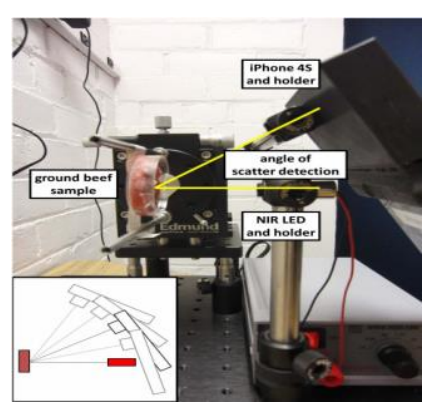

(a)

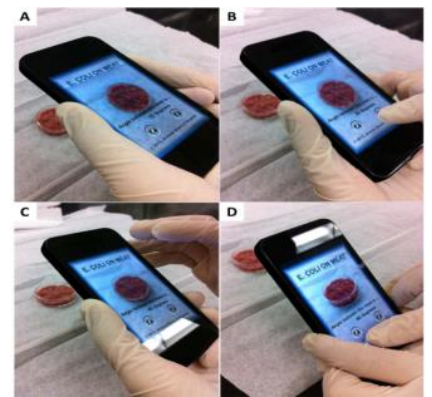

(b)

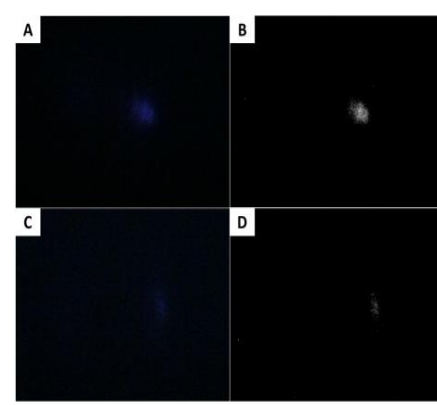

(c)

Figure 4 (a) This bench top system contains an iPhone 4S, NIR LED and ground beef sample placed on the handle, (b) smartphone-based detection system at an angle of (A) $15^{\circ}$ (B) $30^{\circ}$ (C) $40^{\circ}$ (D) $60^{\circ}$, (c) Figure (A) shows raw image (B) Processed image of (A) at higher light intensity using iPhone camera while Figure (C) shows a raw image (D) Processed image of (C) at lower light intensity using an iPhone camera

In addition, according to (Zhu et al., 2012) smartphones integrated with glass capillaries, filters and external lenses which were then connected to a light plastic case where these glass caps function as microfluidic channels. This system is easy to use, compact and cost effective for E.coli detection. A study by (Zhu, et al, 2012) showed that Gold nanoparticles (AuNPs) were used to detect E.coli bacterial concentrations as well as smartphone APP lighting to monitor colour changes on AuNPs. If E.coli bacteria is detected, the colour of AuNPs will change from blue to red and this APP sensor is used to calculate the number of E.coli bacteria. This app contains 2 menus, colour scan and concentration analysis to detect RGB values. The focal length between the sample and the smartphone is maintained at $5 \mathrm{~cm}$ for high precision and accuracy. 
$\mathrm{Cu}$ reacts with Gold nanoparticles (AuNPs) to trigger colour changes that can be detected by using apps and smartphones. This technique according to (Zheng, et al, 2019) and (Mou, et al, 2019) is capable of detecting E.coli as low as $50 \mathrm{CFU} / \mathrm{ml}$ in 1 hour.

\section{SMARTPHONE AND PAPER MICROFLUIDICS}

Detection of E.coli using smartphone and paper microfluidics was introduced by (Park and Yoon, 2015). Microfluidics are made up of three channels namely low detection, high detection and conjugated beads as shown in Figure 5. This paper chip can be used by putting it in a water sample or this water sample will be inserted into a pipette and then passed onto a paper chip. The detection rate of a single cell is 90 seconds and this method combines the smartphone applications and gyro-sensor functionality found in smartphones. This paper chip is placed at a distance of $9 \mathrm{~cm}$ from the smartphone camera and the image will be captured using apps installed inside the smartphone. This app is built using $\mathrm{C}$ objective programming compatible with iPhone. This image will be captured in auto mode using internal gyro sensor. The gyro sensor is used, as it can sense rotational motion and identify orientation changes. This system for gyro sensors is present in the iPhone's smartphone (Umek, et al, 2015). The drawback of using microfluidics paper technology is that E.coli results are not available in real time. Images captured using a smartphone need to be transferred to a laptop for analysis purposes using Matlab software. The code algorithm will convert this colour image to the green image and will be divided into 3 channels. E.coli will be detectable based on average green pixel intensities.
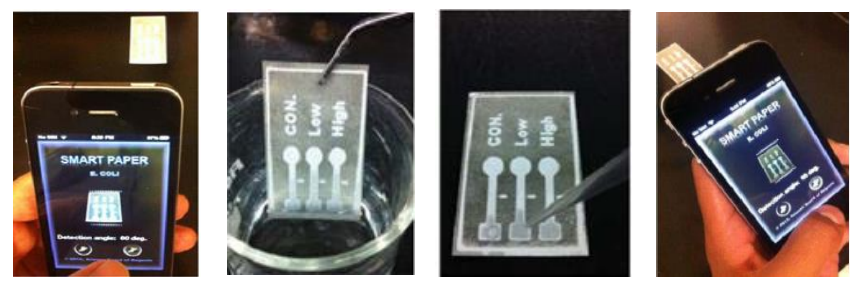

Figure 5: Step to use smartphone and paper microfluidics to test water samples 


\section{FIBER OPTICS}

Figure 6 shows the process of detecting E.coli using fiber optics by mixing the Colilert reagent in $100 \mathrm{~mL}$ water sample. This automatic detection of E.coli can be detected within 16 hours and the bacterial recognition rate is at 1 bacteria per $100 \mathrm{~mL}$ of water sample (1CFU / 100mL). Among the hardware used are raspberry pi, CMOS camera, optical fibers, plexiglas and UV LEDs. Matlab software and cross-correlation methods are used to process raw image. E.coli can be detected if the intensity exceeds $20 \%$ after the first 75 minutes. A study by (Tok, et al, 2019) showed that it can saves time compared to visual inspection, does not require trained staff, reduces counting error for calculating E.coli and 40 samples of water can be analyzed simultaneously.
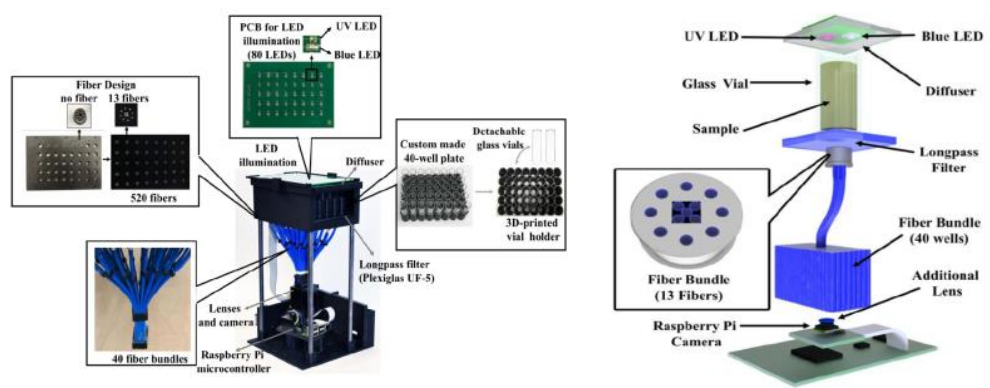

Figure 6: Automatic detection of E.coli using fiber optics and raspberry pi [20]

\section{CONCLUSION}

The issue with using naked eye to detect E.coli is that it takes a long time and needs qualified staff to handle the chemical testing. Using SPR technology, smartphones, biosensors, paper microfluidics and fiber optics making the E.coli detection process easier, using less samples, automated monitoring and analysis, does not require qualified staff, save time, portability and experimentation samples are not confined to the lab area but can be done on-site. The technological advances in the identification of E.coli are useful because E.coli can be identified as soon as possible and the risk to the public is reduced. Therefore, imaging methods for detecting the presence of E.coli are proposed for integration with incubators. This is because the water or food sample needs to be incubated for the process of growing the culture. The image processing can be paired with controllers such as Arduino and Raspberry pi to control the incubator at 
optimal temperature and humidity, mixture between sample and reagent and finally image processing algorithm can be used to analyse the presence of E.coli in water or food samples.

\section{ACKNOWLEDGEMENT}

This research was funded by a T-ARGS research grant Department of Polytechnic and Community College Education, Ministry of Higher Education of Malaysia.

\section{REFERENCES}

Wisuthiphaet, N., Yang, X., Young, G. M., \& Nitin, N. (2019). Rapid detection of Escherichia coli in beverages using genetically engineered bacteriophage T7. AMB Express, 9(1). doi: 10.1186/s13568-019-0776-7.

Dunca, A.-M. (2018). Water Pollution and Water Quality Assessment of Major Transboundary Rivers from Banat (Romania). Journal of Chemistry, 2018, 1-8. doi: 10.1155/2018/9073763.

Odonkor, Stephen. (2013). E coli as an indicator of bacteriological quality of water: An overview. Microbiology research. 4. doi: 10.4081/mr.2013.e2.

Lim, J. Y., Yoon, J., \& Hovde, C. J. (2010). A Brief Overview of Escherichia coli O157:H7 and Its Plasmid O157. Journal of Microbiology and Biotechnology, 20(1), 5-14. doi: 10.4014/jmb.0908.08007.

Xu, Dandan \& Huang, Xiwei \& Guo, Jinhong \& Ma, Xing. (2018). Automatic Smartphone-based Microfluidic Biosensor System at the Point of Care. Biosensors and Bioelectronics. doi: 10.1016/j.bios.2018.03.018.

Umek, A., Kos, A., \& Tomaic, S. (2015). Validation of Smartphone Gyroscopes for Angular Tracking in Biofeedback Applications. 2015 International Conference on Identification, Information, and Knowledge in the Internet of Things (IIKI). doi: 10.1109/iiki.2015.70 
Rettner, R., 1st Death Tied to Outbreak of E. Coli in Romaine Lettuce - How It Kills. (2018). Retrieved from https://www.livescience.com/62466-how-e-colikills.html.

Almadidy, A., Watterson, J., Piunno, P. A., Raha, S., Foulds, I. V., Horgen, P. A., Krull, U. (2002). Direct selective detection of genomic DNA from coliform using a fiber optic biosensor. Analytica Chimica Acta, 461(1), 37-47. doi:10.1016/s0003-2670(02)00243-x.

Ahmed, M. O., Almshawt, N. F., \& Elnageh, H. R. (2017). Diarrheagenic Escherichia coli $\mathrm{O} 157$ from Libya: recent perspectives and challenges. Journal of Public Health in Africa, 8(1). doi: 10.4081/jphia.2017.685.

Sukhumungoon, P. (2015). Incidence of Escherichia coli O157:H7 in Thailand. Sains Malaysiana, 44(2), 261-267. doi: 10.17576/jsm-2015-4402-14.

Kabiru, L., Bello, M., Kabir, J., Grande, L., \& Morabito, S. (2015). Detection of Pathogenic Escherichia coli in Samples Collected at an Abattoir in Zaria, Nigeria and at Different Points in the Surrounding Environment. International Journal of Environmental Research and Public Health, 12(1), 679-691. doi: 10.3390/ijerph120100679.

Jang, J., Hur, H.-G., Sadowsky, M., Byappanahalli, M., Yan, T., \& Ishii, S. (2017). Environmental Escherichia coli: ecology and public health implications-a review. Journal of Applied Microbiology, 123(3), 570-581. doi: 10.1111/jam.13468.

Wang, Y., \& Alocilja, E. C. (2015). Gold nanoparticle-labeled biosensor for rapid and sensitive detection of bacterial pathogens. Journal of Biological Engineering, 9(1). doi: 10.1186/s13036-015-0014-z.

Clark, S. A. (2001). Rapid detection assays for food and water. Cambridge: Royal Society of Chemistry. 
Bajwa, A., Tan, S., Mehta, R., \& Bahreyni, B. (2013). Rapid Detection of Viable Microorganisms Based on a Plate Count Technique Using Arrayed Microelectrodes. Sensors, 13(7), 8188-8198. doi: 10.3390/s130708188.

Disotell, T. R. (2017). Polymerase Chain Reaction (PCR), Quantitative/Real-Time PCR (qPCR), Reverse Transcriptase PCR (RT-PCR). The International Encyclopedia of Primatology, 1-2. doi: 10.1002/9781119179313.wbprim0483.

Babaie, Z., Isfahani, B., Fazeli, H., Poursina, F., Moghim, S., \& Rouzbahani, M. (2017). Evaluation of Polymerase Chain Reaction for Detecting Coliform Bacteria in Drinking Water Sources. Advanced Biomedical Research,6(1), 130. doi: $10.4103 / 2277-9175.216783$.

Zordan, M. D., Grafton, M. M., Acharya, G., Reece, L. M., Cooper, C. L., Aronson, A. I., Leary, J. F. (2009). Detection of pathogenicE. coliO157:H7 by a hybrid microfluidic SPR and molecular imaging cytometry device. Cytometry Part A, 75A(2), 155-162. doi:10.1002/cyto.a.20692.

Berney, M., Hammes, F., Bosshard, F., Weilenmann, H., \& Egli, T. (2007). Assessment and Interpretation of Bacterial Viability by Using the LIVE/DEAD BacLight Kit in Combination with Flow Cytometry. Applied and Environmental Microbiology, 73(10), 3283-3290. doi:10.1128/aem.02750-06.

Lahtinen, S. J., Ouwehand, A. C., Reinikainen, J. P., Korpela, J. M., Sandholm, J., \& Salminen, S. J. (2006). Intrinsic Properties of So-Called Dormant Probiotic Bacteria, Determined by Flow Cytometric Viability Assays. Applied and Environmental Microbiology, 72(7), 5132-5134. doi:10.1128/aem.02897-05.

Liang, P., Park, T. S., \& Yoon, J. (2014). Rapid and reagentless detection of microbial contamination within meat utilizing a smartphone-based biosensor. Scientific Reports, 4(1). doi:10.1038/srep05953. 
Zhu, H., Sikora, U., \& Ozcan, A. (2012). Quantum dot enabled detection of Escherichia coli using a cell-phone. The Analyst, 137(11), 2541. doi: 10.1039/c2an35071h.

Zheng, L., Cai, G., Wang, S., Liao, M., Li, Y., \& Lin, J. (2019). A microfluidic colorimetric biosensor for rapid detection of Escherichia coli O157:H7 using gold nanoparticle aggregation and smart phone imaging. Biosensors and Bioelectronics, 124-125, 143-149. doi: 10.1016/j.bios.2018.10.006.

Mou, X.-Z., Chen, X.-Y., Wang, J., Zhang, Z., Yang, Y., Shou, Z.-X. Li, Y.-Q. (2019). Bacteria-Instructed Click Chemistry between Functionalized Gold Nanoparticles for Point-of-Care Microbial Detection. ACS Applied Materials \& Interfaces, 11(26), 23093-23101. doi: 10.1021/acsami.9b09279.

Park, T. S., \& Yoon, J.-Y. (2015). Smartphone Detection of Escherichia coli From Field Water Samples on Paper Microfluidics. IEEE Sensors Journal, 15(3), 19021907. doi: 10.1109/jsen.2014.2367039.

Tok, S., Haan, K. D., Tseng, D., Usanmaz, C. F., Koydemir, H. C., \& Ozcan, A. (2019). Early detection of E. coli and total coliform using an automated, colorimetric and fluorometric fiber optics-based device. Lab on a Chip, 19(17), 2925-2935. doi: $10.1039 / \mathrm{c} 91 \mathrm{lc} 00652 \mathrm{~d}$. 